by the relief of symptoms but by longer survival, clearly there is much room for improvement.

Accurate assessment of the prognosis in the individual patient is the key to successful management. The disease has a wide range of expression from the clinical silence of latent carcinoma to an aggressive metastatic cancer. The "TNM" classification of the International Union against Cancer (UICC) provides a worldwide basis for clinical staging. Its accuracy can be increased by the newer techniques for identifying metastases, such as lymphangiography, skeletal scintigraphy, and bone marrow biopsy. Gleason's studies make a strong case for histological grading as a powerful guide to prognosis. ${ }^{34}$ The Scandinavian school's experience of cytology has shown how transrectal needle aspiration of a primary tumour can be used in diagnosis and prognosis as well as in assessing the response to treatment. ${ }^{5}$

The levels of follicle-stimulating hormone, prolactin, and luteinising hormone may be important criteria by which to judge response to treatment, but their interpretation still presents problems. ${ }^{6}$ Likewise, determining plasma protein profiles, including those of the steroid transport proteins, could be a valuable tool once an appropriate array of individual proteins has been chosen, though this approach too is bedevilled by the powerful pharmacological effects of hormone changes on protein control mechanisms. The search for receptors for steroid hormone in carcinoma of the prostate is interesting many biologists, but so far their studies have not succeeded in giving clinicians a scientific basis for endocrine treatment.

Chemotherapy presents a special challenge, as patients with prostatic cancer are generally elderly and often have widespread lesions in the bone marrow. But the rather small number of patients in the average urological practice, perhaps 30 a year, and their diverse subsets make it impossible to achieve progress without uniting the resources of many urologists. The subsets depend not only on the anatomical distribution of the cancer but on its biological behaviour. Lymphangiography has shown that spread to the lymph nodes does not always lead on to the lethal forms of the disease. ${ }^{7}$ Only time will tell if we can eventually pick out those tumours whose spread is restricted to lymph nodes from those that disseminate to the bones. Apart from relieving urinary obstruction a wait-and-see policy might be justified for the former and more aggressive treatment reserved for the latter.

${ }^{1}$ Bailer, J C, III, and Byar, D C, Cancer, 1970, 26, 257.

2 Blackard, C E, Cancer Chemotherapy Reports, 1975, 59, 225.

3 Gleason, D F, Cancer Chemotherapy Reports, 1966, 50, 125.

4 Gleason, D F, Mellinger, G T, and the Veterans Administration, fournal of Urology, 1974, 111, 58.

${ }^{5}$ Esposti, P L, Scandinavian fournal of Urology and Nephrology, 1971, 5, 199.

${ }^{6}$ Harper, M E, et al, Acta Endocrinologica, 1976, 81, 409.

7 Paxton, R M, et al, British Medical fournal, 1975, 1, 120.

\section{Psoriatic arthritis: to lump or to split?}

Rheumatologists differ in their desire to describe in detail or to merge into a large comprehensive disease pattern. Is he a lumper or a splitter? used to be asked of certain great men. In the prewar years lumping was the order. All inflammatory arthropathies were variants of rheumatoid arthritis, and disorders such as ankylosing spondylitis, different in almost every respect, got lost in the vast maw of the commoner disorder. In the classification of rheumatic diseases proposed in 1957 by the International League against Rheumatism psoriatic arthritis, ankylosing spondylitis, and Reiter's (Brodie's) disease were considered merely variants or special varieties of rheumatoid arthritis, though this was rectified a few years later. This led to a neglect of a proper study of these disorders. Polymyalgia rheumatica, when considered a variant of rheumatoid arthritis occurring in the elderly, attracted little or no attention until Barber ${ }^{1}$ in 1957 described it as a disease of unknown aetiology and not a type of rheumatoid arthritis under the somewhat unsatisfactory title of polymyalgia rheumatica. Only then did clinicians give it the attention and study that it deserved. In the last 30 years or so the splitters seem to have added far more to our knowledge of the rheumatic disorders than the lumpers.

When Gibberd ${ }^{2}$ reviewed 915 patients with rheumatoid arthritis who had attended the rheumatism unit at Westminster Hospital between 1950 and 1961 he found that only one of 406 classical seropositive rheumatoid patients with erosive bone disease had psoriasis as compared with 14 of the less severe seronegative cases. By this time several workers ${ }^{3-5}$ had pointed out the difference between the two disorders, and psoriatic arthritis was being considered a separate disease in its own right. What is the evidence for and against this today?

Recently Roberts and colleagues ${ }^{6}$ have reviewed 227 patients with psoriasis and various forms of arthritis. Five had gout and 54 osteoarthritis or some other non-inflammatory rheumatic complaint, but 168 had an inflammatory arthritis. Of these 94 were followed up for over 10 years. These workers found an arthritis indistinguishable from rheumatoid disease in $78 \%$, distal joint arthritis in $17 \%$, and a deforming arthritis in $5 \%$. In a large majority of cases skin lesions preceded the arthritis, but in $16 \%$ the arthritis came first; rarely did the two start together. Apart from a small group of patients with deforming disease the arthritis was mild as judged by time off work and number of hospital admissions. Deterioration, clinical or radiological, occurred only in a minority of patients. The onset of arthritis was acute in almost half the patients, the peak age at onset being 36-45 years. Although the sheep cell agglutination test was negative in most, $16 \%$ were persistently and $10 \%$ variably positive. Subcutaneous rheumatoid nodules were present in only five cases, all in the group indistinguishable from rheumatoid arthritis, and all five had positive serology. These five would appear to be cases of true rheumatoid arthritis with coincidental psoriasis, and the same may well be true of the others in this group with positive serological tests for rheumatoid factor. Nevertheless, the fact remains that over a 10-year follow-up most remained seronegative. This could mean that either they were cases of mild rheumatoid disease who had not progressed to seropositivity, or they had a different disorder-psoriatic arthritis.

The diagnosis of psoriatic arthritis is usually considered when (1) the seronegative nodule-free patient has psoriasis, however mildly, and (2) the disease is patchy in distribution, less symmetrical and less aggressively progressive, and has attacked the terminal interphalangeal joints of the hands in addition to other joints. The present study confirms this up to a point, but also shows that the largest group were indistinguishable from rheumatoid arthritis though remaining in most cases seronegative, the group with distal joint arthritis being much smaller. In this last group the appearance was more characteristic of psoriatic arthritis as described above, but even here one patient was constantly and one intermittently weakly seropositive.

In psoriatic arthritis the prevalence of bilateral sacroiliitis is 
about $20 \%,^{78}$ and Wright and colleagues ${ }^{9}$ have recently discussed in detail the seronegative spondylarthritides, one of which is associated with psoriasis. Hence psoriasis, a common disorder, may coexist with rheumatoid arthritis, another common disorder, but is more often associated with an apparently identical arthritis which remains seronegative and is less aggressive and progressive. It may also present in the familiar distal form and also as a seronegative spondylarthritis, and sometimes as both. At the moment, therefore, there is enough evidence for us to accept psoriatic arthritis as an entity distinct and apart from rheumatoid arthritis, even though the manifestations are often similar or identical and even though patients with classical rheumatoid arthritis may also have coexistent psoriasis. Patients with psoriatic arthritis should by definition be seronegative and free from rheumatoid nodules. By splitting this disorder off from rheumatoid arthritis much more has been learnt about it, as these workers have clearly shown.

${ }^{1}$ Barber, H S, Annals of the Rheumatic Diseases, 1957, 16, 230.

2 Gibberd, F B, Acta Rheumatologica Scandinavica, 1965, 11, 62.

3 Vilanova, X, and Pinol, J, Rheumatism, 1951, 7, 197.

4 Wright, V, Annals of the Rheumatic Diseases, 1956, 15, 348.

${ }^{5}$ Coste, F, et al, Revue du Rhumatisme et des Maladies Ostéo-Articulaires, $1958,25,75$.

${ }^{6}$ Roberts, M E T, et al, Annals of the Rheumatic Diseases, 1976, 35, 206.

7 Read, W B, Heiskell, C L, and Becker, S W, Archives of Dermatology, $1961,83,653$.

8 Wright, V, Annals of the Rheumatic Diseases, 1961, 20, 123.

9 Wright, V, Sturrock, R D, and Dick, W C, in Recent Advances in Rheumatology, Part 2, eds W W Buchanan and W C Dick, p 193. London, Churchill Livingstone, 1976.

\section{Cigarette smoking in pregnancy}

Cigarette smoking often constitutes an addiction or provides a form of escape from anxiety about personal problems. But smoking in pregnancy presents a special case. Firstly, it is a danger to the child as well as to the mother. Secondly, since pregnancy is of limited duration a woman who cannot be persuaded to give up smoking may accept a substitute at least while she is pregnant. It would be an advance if her craving could be met by a cigarette free from dangers to the baby. Accordingly, workers have tried to identify the harmful components of tobacco smoke.

Carbon monoxide, nicotine, and cyanides are the factors which seem most likely to have ill effects on the fetus. Studies of carbon monoxide ${ }^{1-3}$ have indicated that the increase in carboxyhaemoglobin may deprive the fetus of adequate oxygen. Epidemiological evidence suggests that the type of baby liable to be harmed by the mother's smoking is the one already at risk for some other reason. Nevertheless, analysis of oestriol excretion levels has suggested that hypoxia is not the main influence on retardation of fetal growth. ${ }^{4}$

Large doses of nicotine injected into pregnant rats reduce birth weight. ${ }^{5}$ Suzuki et $a l^{6}$ injected nicotine into pregnant rhesus monkeys and concluded that the concentration produced by maternal smoking would reduce the fetal oxygenation. In man Gennser $\mathrm{et}^{\mathrm{al}} \mathrm{l}^{7}$ compared the effects of nicotine in chewing-gum at two strengths, tobacco cigarettes, and nontobacco cigarettes. They measured changes in fetal respiratory movements and maternal blood nicotine and carboxyhaemoglobin levels. The tobacco cigarettes and both strengths of nicotine chewing-gum raised the nicotine level and the tobacco and non-tobacco cigarettes raised the carboxyhaemoglobin level, but for each measurement the tobacco cigarette had the greatest effect. Fetal breathing was depressed with the tobacco cigarette and slightly less so with the nicotine chewing-gum. A more recent study has confirmed that fetal breathing movements are reduced in association with cigarette smoking in normal and abnormal pregnancies. ${ }^{8}$ The reduction was related to the rise in maternal plasma nicotine levels. Nonnicotine (herbal) cigarettes produced similar rises in carboxyhaemoglobin to those caused by tobacco cigarettes but without a reduction in fetal breathing. These studies are helpful in dissociating the influence of nicotine from that of carbon monoxide in regard to this particular effect. Nevertheless, it does not follow that the effect on fetal respiration is of practical importance, though circumstantial evidence is accumulating that it may be. ${ }^{8}$

Thiocyanate concentrations are raised in the cord blood of infants born to smoking mothers, ${ }^{9}$ while vitamin $B_{12}$ levels are lower in smokers. This may reflect a disorder of cyanide detoxification $^{10}$ related to the cyanide of the cigarette smoke. The lowered appetite which is a recognised concomitant of smoking may be responsible for a lower maternal calorific intake and hence lower fetal weight, but a review of the evidence in 1973 did not suggest that this mattered." Since then, however, other workers have suggested that impaired gain in maternal weight may be of importance. ${ }^{11}$

What can be done practically? Until the metabolites which are dangerous to the fetus have been identified with certainty, efforts to substitute an alternative to tobacco cigarettes seem premature. Attempts to persuade the mother to reduce or preferably give up smoking offer the best prospect, though it is worth bearing in mind that a patient deprived of one satisfaction for her craving may try to resort to another.

Smoking is best stopped before conception, but there is evidence that stopping in the first two months of pregnancy reduces the hazard. ${ }^{13}$ Encouraging the mother to increase her food intake may be worth trying, but excessive weight gain may be harmful, and at present a controlled trial of this approach seems called for. ${ }^{12}$ It is mothers in the lower social classes and with a poor obstetric performance whose babies are particularly at risk from smoking, and they might well be guided to special clinics so that maximal effort could be concentrated on them. Whatever his detailed approach, the obstetrician must take pains not to be so critical as to drive the patient away from the antenatal care which he offers but show compassionate understanding of a common human frailty.

1 Younoszai, M K, Kacic, A, and Haworth, J C, Canadian Medical Association fournal, 1968, 99, 197.

2 Astrup, P, et al, Lancet, 1972, 2, 1220

${ }^{3}$ Cole, P V, Hawkins, L H, and Roberts, D, fournal of Obstetrics and Gynaecology of the British Commonwealth, 1972, 79, 782.

- Targett, C S, et al, Fournal of Obstetrics and Gynaecology of the British Commonwealth, 1973, 80, 815.

${ }^{5}$ Public Health Services, The Health Consequences of Smoking. A Report of the Surgeon General, 1973. Department of Health, Education and Welfare, DHEW Publication No. (HMS) 73-8704, 1973.

${ }^{6}$ Suzuki, K, et al, American fournal of Obstetrics and Gynecology, 1971, 111, 1092 .

7 Gennser, G, Marsal, K, and Brantmark, B, American fournal of Obstetrics and Gynecology, 1975, 123, 861 .

${ }^{8}$ Manning, F A, and Feyerabend, C, British fournal of Obstetrics and Gynaecology, 1976, 83, 262.

9 Andrews, J, Fournal of Obstetrics and Gynaecology of the British Commonwealth, 1973, 80, 810 .

10 McGarry, J M, and Andrews, J, British Medical fournal, 1972, 2, 74.

${ }^{11}$ Rush, D, Fournal of Obstetrics and Gynaecology of the British Commonwealth, 1974, 81, 746.

12 Davies, D P, et al, Lancet, 1976, 1, 385.

13 Butler, N R, Goldstein, H, and Ross, E M, British Medical fournal, 1972, 2, 127. .}

